\title{
BANCO DE DADOS DE COCCOIDEA (INSECTA: HEMIPTERA: STERNORRHYNCHA) DA COLEÇÃO ENTOMOLÓGICA ADOLPH HEMPEL, INSTITUTO BIOLÓGICO.
} FRANCO, K.P.A.C. ${ }^{1,2^{*}}$; IDE, S. ${ }^{1}$ Instituto Biológico, Centro de Pesquisa de Sanidade Vegetal, Laboratório de Entomologia Geral, Av. Cons. Rodrigues Alves, 1252, CEP 04014-900, São Paulo, SP, Brasil. 2Universidade Presbiteriana Mackenzie, São Paulo, SP, Brasil. E-mail: kayhecury@gmail.com. Database of Coccoidea (Insecta: Hemiptera: Sternorrhyncha) of the Coleção Entomológica Adolph Hempel, Instituto Biológico.

O acervo de Coccoidea (Insecta: Hemiptera: Sternorrhyncha) da Coleção Entomológica Adolph Hempel, Instituto Biológico (São Paulo) é considerado o mais importante do Brasil, pela quantidade de tipos. É resultado do trabalho de pesquisadores como A. Hempel, H.S. Lepage, J.P. da Fonseca e O. Giannotti, que dedicaram grande esforço para sua formação. A Coleção inclui material em 930 lâminas, 1.000 tubos de vidro e em torno de 340 envelopes. O banco de dados é composto por sete tabelas com informações sobre os dados de coleta, hospedeiros e publicações e conta com 142 registros de Coccoidea. Foram incluídos e/ou atualizados dados referentes aos seguintes táxons: [ACLERDIDAE] - Aclerda santensis Hempel, 1937 e A. campinensis Hempel, 1934; [Asterolecanitdat] - Asterolecanium bambusae Boisduval, 1869, A. bondari Lepage, 1940, A. miliaris (Boisduval, 1869), A. sanbernardense Hempel, 1937, A. pustulans (Cockerell, 1892), A. viridulum Cockerell, 1902, e Asterolecanium sp.; [CERococcidAe] - Cerococcus parahybensis Hempel, 1927 e Cerococcus sp.; [LECANODIASPIDIDAE] - Lecanodiaspis rugosa (Hempel, 1900) e Lecanodiaspis sp.; [CoccIDAE] - Akermes riograndensis Hempel, 1932, Alecanochiton marquesi Hempel, 1921, Alichtensia attenuata (Hempel, 1900), Anopulvinaria cephalocarinata Fonseca, 1972, Coccus hesperidum Linnaeus, 1758, C. viridis (Green, 1889), Coccus sp., Eucalymnatus delicatus Hempel, 1937, E. itanhaensis Mendes, 1931, Eucalymnatus sp., Eulecanium sp., Inglisia australis Hempel, 1937, Mesolecanium mayteni (Hempel, 1900), M. obscurum (Hempel, 1900), M. planum Hempel, 1932, M. pseudosemen (Cockerell, 1895), Milviscutulus mangiferae (Green, 1889), Neolecanium silveirai (Hempel, 1900), Neolecanochiton grevilleae Hempel, 1932, Parakermes brasiliensis Fonseca, 1973, Parapulvinaria cassariae Fonseca, 1969, Parasaissetia nigra (Nietner, 1861), Parthenolecanium perlatum (Cockerell, 1898), Pendularia pendens Fonseca, 1927, Platinglisia noacki Cockerell, 1899, Protopulvinaria longivalvata Green, 1909, Protopulvinaria sp., Pseudalichtensia brasiliae Hempel, 1928, Pseudokermes nitens (Cockerell, 1895), P. palmae Hempel, 1937, Pseudokermes sp., Pseudophilippia lanigera (Hempel, 1900), Pulvinaria convexa (Hempel, 1900), P. depressa Hempel, 1900, P. eryngii Fonseca, 1973, P. ficus Hempel, 1900, P. flavescens Brèthes in Massini \& Brèthes, 1918, P. justaserpentina Fonseca, 1973, P. paranaenses Hempel, 1928, Pulvinaria sp., Saissetia coffeae (Walker, 1852), S. discoides (Hempel, 1900), S. minensis Hempel, 1932, S. oleae oleae (Olivier, 1791), S. reticulata (Cockerell, 1895), S. socialis Hempel, 1932, Stictolecanium ornatum (Hempel, 1900), Tectopulvinaria albata Hempel, 1900, Toumeyella nectandrae Hempel, 1929, T. paulista Hempel, 1932, Vinsonia stellifera (Westwood, 1871) e Lecaniinae indet.; [ERIOcocCIDAE] - Chaetococcus bambusae (Maskell, 1893), Eriococcus araucariae Maskell, 1879, E. campinensis Hempel, 1937, E. coccineus Cockerell, 1894, E. lanatus Hempel, 1932, E. piptandeniae Hempel, 1937, Eriococcus sp., Pseudotectococcus anonae Hempel, 1934 e $P$. rolliniae Hodgson \& Gonçalves in Hodgson, 2004; [PsEudococcIDAE] - Erium luciliae Hempel, 1932 e [DiASPIDIDAE] - Acutaspis oliveirae (Fonseca \& Lepage, 1942), $A$. scutiformis (Cockerell, 1893), Acutaspis sp., Aonidiella aurantii (Maskell, 1879), Furcaspis aequatorialis Williams \& Miller, 2006, F. bromeliae Hempel, 1932, F. mexicana Williams \& Miller, 2006, F. palmaria Williams \& Miller, 2006, F. paxilliloba Williams \& Miller, 2006, F. plana Hempel, 1937, F. scleroprymna Williams \& Miller, 2006, F. taquarae Fonseca, 1969, Mycetaspis brasiliensis Hempel, 1932 e M. juventinae Lepage \& Giannotti, 1944.

*Bolsista PIBIC/CNPq/IB 